\section{A case of Henoch-Schönlein purpura with cardiopulmonary involvement}

\author{
Guang Ming Tan \\ Division of Cardiology, Department of \\ Medicine and Therapeutics, Prince of \\ Wales Hospital, The Chinese University \\ of Hong Kong, Hong Kong
}

\begin{abstract}
Henoch-Shönlein purpura (HSP) commonly affects the kidney, causing hematuria and Immunoglobulin A type nephropathy. Extrarenal involvements are rare but can be life threatening if missed. Cardiac involvement has been described only in a handful of case reports. Although HSP is predominantly a pediatric disease, it should not be overlooked in older patients presenting with typical triad of symptoms. We report a rare case of HSP with both cardiac of pulmonary systems involvement and his clinical progress
\end{abstract}

\section{Introduction}

Henoch-Shönlein purpura (HSP) is an immunoglobin (Ig) A mediated generalized vasculitic condition of the small vessels commonly affecting children aged 2-11 years old. Ig A immune complex deposition in the vessel walls of the target organs escalating vascular inflammatory mediators such as prostacyclins and endothelins plays a central role in the pathogenesis of HSP. A wide variety of pathogens especially recent streptococcal infection, drugs, and other environmental exposures such as cold might stimulate the production of Ig A and have been associated with HSP. The commonest organ systems affected are the skin, the kidneys, the joints and the gastrointestinal tract, resulting in the classical triad of symptoms including purpura, arthritis, and abdominal pain. Other organ systems such as the lung and the heart can be rarely affected too. The most feared complication of HSP is the development of end-stage renal disease, which occurs in one percent of the patients. ${ }^{1,2}$ Here we report a case of adult onset HSP who developed ST elevation myocardial infarction (STEMI) and pulmonary hemorrhage in the course of his illness.

\section{Case Report}

A 60-year-old patient first sought medical attention because of two weeks history of watery diarrhea, vomiting and colicky abdominal pain. He visited his family physician and was given symptomatic treatment without improvement. One week after his symptom onset, he developed purpuric rash on bilateral lower limbs and joints pain involving multiple sites including bilateral knees and elbow. He did not have other systemic complains such as hematuria, dysuria, ocular problem, or oral ulcers. He is a chronic smoker who has been smoking one pack per day for forty years. While waiting at the clinic, he developed an episode of near-syncope, and electrocardiogram (ECG) on the spot showed hyperacute $\mathrm{T}$ wave over leads V1-V3. ECG repeated 15 minutes later showed evolution into ST elevation (STE) over the aforementioned leads. Patient at that time did not complain angina, shortness of breath or palpitation. He was afebrile, and his vital status was normal. Systemic examination revealed non-blancable palpable purpuric lesions and mild edema around bilateral ankles. Other systems examination were unremarkable.

In view of the acute ECG changes, patient was admitted to Cardiac Care Unit (CCU) after stabilization. Repeat ECG two hour after the initial ECG showed spontaneous evolution of STE . Initial blood test showed deranged renal function (RFT), with creatinine rose to 207 umol/L. Other electrolytes including potassium, magnesium and calcium were normal. Liver function test and amylase was normal. Hemoglobin ( $\mathrm{Hb})$ and platelet level was normal, while white cell count was elevated to 19.2 $10^{9} / \mathrm{L}$, with neutrophils predominant. Troponin T level was elevated to $232 \mathrm{ng} / \mathrm{L}$, while serial creatine kinase level was normal. (CK-MB level was not available as a routine test in our unit). Serum C3 and C4 level was normal. Antinuclear antibodies and Anticardiolipin antibodies were negative. Urine multistix was negative for red blood cell and protein. Stool culture and microscope was negative. First chest X-ray was clear. Bedside echocardiogram performed after admission to CCU showed no regional wall movement abnormality and preserved left ventricular systolic function without significant valvular lesion.

Patient was first managed as acute coronary syndrome and was started on aspirin and subcutaneous low molecular weight heparin (LMWH) in view of the ECG finding and raised troponin T level. Pulse steroid with intravenous methylprednisolone was also started because of the clinical finding of purpuric rash, joints pain, and deranged renal function.
Correspondence: Guang Ming Tan, Division of Cardiology, Department of Medicine and Therapeutics, Prince of Wales Hospital, The Chinese University of Hong Kong, 30-32 Ngan Shing Street, Hong Kong.

Tel.: +852.263.22211 - Fax: +852.263 .21735 .

E-mail: tamgormin@hotmail.com

Key words: Henoch-Shönlein purpura STEMI; vasculitis; pulmonary hemorrhage.

Conflict of interest: the author declares no potential conflict of interest.

Received for publication: 6 February 2015.

Revision received: 29 March 2015.

Accepted for publication: 30 March 2015.

This work is licensed under a Creative Commons Attribution NonCommercial 3.0 License (CC BYNC 3.0).

(C) Copyright G.M. Tan, 2015

Licensee PAGEPress, Italy

Rheumatology Reports 2015; 7:5854

doi:10.4081/rr.2015.5854

Coronary angiogram was performed on the next day and it showed narrowing of the distal Left Anterior Descending (LAD) artery, which resolved on administration of intracoronary nitrate (Figure 1). Coronary artery spasm was suspected to be the culprit of ECG changes, and aspirin and LMWH were taken off.

Five days after admission, patient was noted to have an acute drop of $\mathrm{Hb}$ from $12 \mathrm{~g} / \mathrm{L}$ to $9 \mathrm{~g} / \mathrm{L}$, without obvious symptoms of bleeding. Repeated CXR showed increased bilateral lung infiltrates, and CT thorax showed bilateral interlobular septal thickening and groundglass changes suggestive of diffuse pulmonary hemorrhage. Bronchoscopy was performed and showed pulmonary hemorrhage with progressive bloody effluent from bilateral bronchial tree, which was suspected to be related to the underlying vasculitic process and the concurrent usage of LMWH. Skin biopsy from lower limb purpuric rash showed leucocytoclastic vasculitis with positive IgA2+ staining on immunoflurescein study (Figure 2), which confirmed the diagnosis of HSP. Patient was continued on three more days of pulse steroid. His symptoms improved after treatment, and the purpuric rash subsided gradually. His renal function normalized, and repeated CXR showed clearing of bilateral lung infiltrates. He was discharged on a maintenance dose of prednisolone of $50 \mathrm{mg} /$ day and rheumatologist planned to give him a course of monthly IV cyclophosphamide infusion. 


\section{Discussion and Conclusions}

HSP commonly affects the kidney, causing hematuria and IgA type nephropathy. Extrarenal involvements are rare but can be life threatening if missed. Cardiac involvement has been described only in a handful of case reports. Atrioventricular block, arrhythmias, autoimmune myocarditis and STEMI are some of the reported cardiac manifestations of HSP. ${ }^{3-8}$ Pulmonary hemorrhage is also a rare complication of HSP. ${ }^{9,10}$ To our knowledge, this is the first to report the involvement of both cardiac of pulmonary systems in HSP.

The prompt normalization of ST segment change in our patient suggested of transient ischemic of the myocardium, and one possible mechanism can be coronary artery spasm precipitated by vasculitic process which damages the coronary vessels endothelium and releases vasoconstrictive substances from those cells, such as endothelin-1 which is known to have potent vasoconstrictive actions. ${ }^{11}$ Such phenomenon was observed in several other vasculitic conditions, and was reviewed in several case reports. ${ }^{12-14}$ Another postulated mechanism of the myocardial ischemia in our patient could be related to the acute arterial thrombosis that was associated with the presence of antiphospholipid antibodies that were sometimes found in patients with HSP. ${ }^{15}$ The antiphospholipid antibodies however was negative in our patient.

In our patient, coronary angiogram was performed to exclude coronary artery stenosis as our patient has multiple risk factors for atherosclerosis, and the angiogram demonstrated coronary artery spasm relieved by nitrate. To our knowledge, ours is the first report to document, angiographically, the vaso-spastic process in HSP patient. Other causes of acute coronary symdrone with normal coronary arteries are: ${ }^{12}$ coronary artery spasm; coronary artery embolism; coronary artery anomaly; pericarditis; myocarditis; cardiomyopathy; aortic stenosis; aortic dissection; pulmonary embolism; syndrome X.

The differential diagnosis of a patient with cutaneous purpura and myocardial infarction can be challenging. However, with the addition of other organ involvement including the kidney, lung and joints, one should suspect a systemic cause, in particular vasculitis of small to medium sized vessels. Although HSP is predominantly a pediatric disease, it can affect adult too, and should not be overlooked in patients passed adolescent year but presenting with typical triad of symptoms. While skin biopsy or tissue biopsy of the organ involved is often required to establish the final diagnosis, this can be time consuming and should not delay treatment if clinical suspicion is high. As with most vasculitic condition, the initial treat- ment for patients suffering from HSP with multi-organ involvement is high dose steroid followed by immunosuppressive therapy, as in our patient. ${ }^{13}$ Although there is evidence on the effectiveness of steroid in treating abdominal pain and joint pain in HSP, there is no good evidence demonstrating the effectiveness of long term steroid or other immunosuppressive therapy in the treatment or prevention of
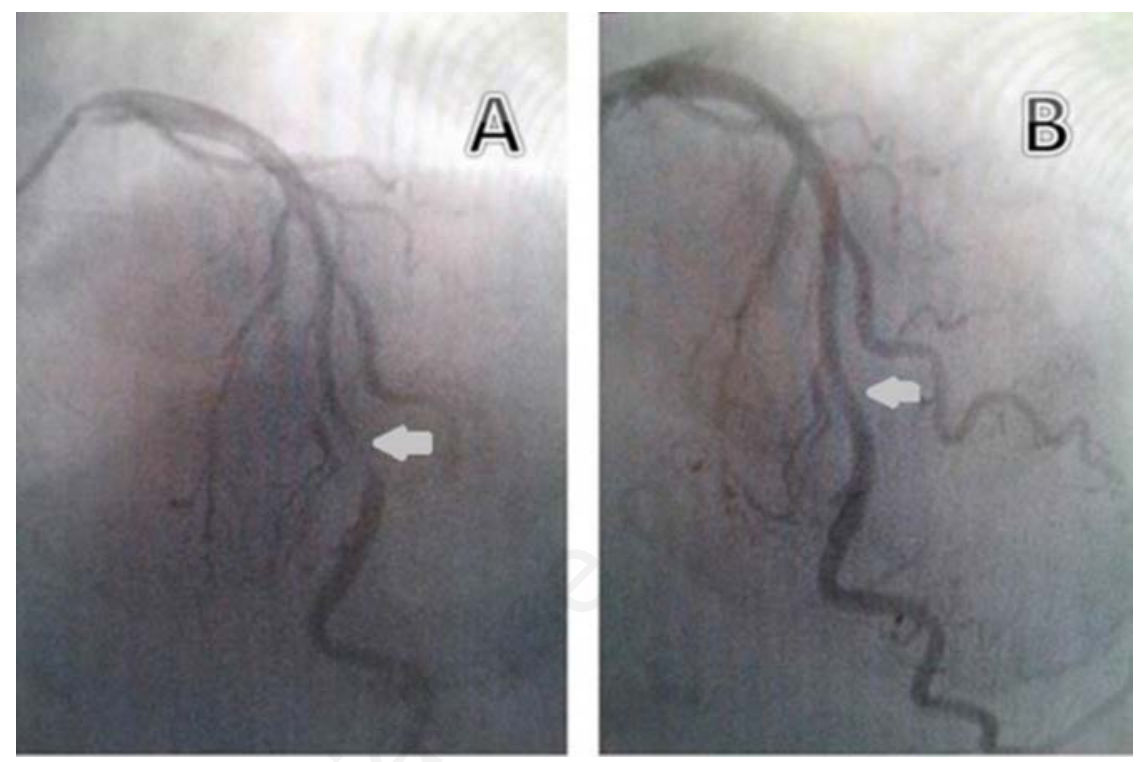

Figure 1. Arrows showing severe spasm of the mid-left anterior descending artery (A), which was relieved after nitrocane injection (B).

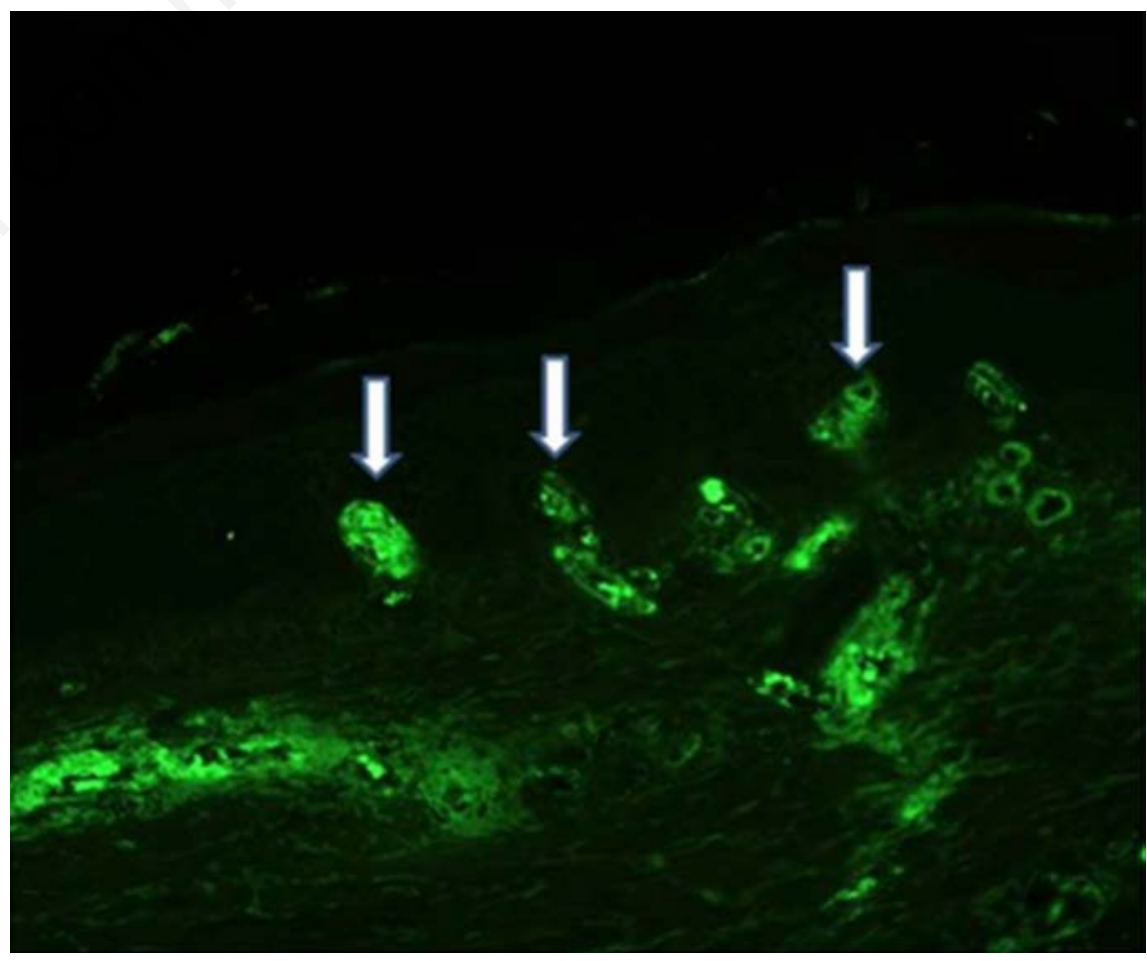

Figure 2. Direct immunofluorescence staining of the skin using anti-immunoglobulin A antibody showing perivascular IgA deposition (arrow) in the superficial capillaries. 
well with no sequela observed so far.

In conclusion, we presented a case of adult onset HSP associated with STEMI and pulmonary hemorrhage from underling vasculitic processes. Although diagnostically challenging, this case illustrated the importance of systemic enquiry and integrated clinical approach.

\section{References}

1. Saulsbury FT. Clinical update: HenochSchönlein purpura. Lancet 2007;369:976-8.

2. Saulsbury FT. Henoch-Schönlein purpura. Curr Opin Rheumatol 2010;22:598-602.

3. Lecutier MA. A case of the SchönleinHenoch syndrome with myocardial necrosis. J Clin Pathol 1952;5:336-8.

4. Abdel-Hadi 0, Greenstone MA, Hartley RB, et al. Myocardial infarction - a rare complication in Henoch-Schönlein purpura. Postgrad Med J 1981;57:390-2.

5. Agraharkar M, Gokhale S, Le L, et al. Cardiopulmonary manifestations of Henoch-Schönlein purpura. Am J Kidney Dis 2000;35:319-22.
6. Polizzotto MN, Gibbs SD, Beswick W, et al. Cardiac involvement in Henoch-Schönlein purpura. Intern Med J 2006;36:328-31.

7. Güven H, Ozhan B, Bakiler AR, et al. A case of Henoch-Schönlein purpura and rheumatic carditis with complete atrioventricular block. Eur J Pediatr 2006;165:395-7.

8. Lutz HH, Ackermann T, Krombach GA, et al. Henoch-Schönlein purpura complicated by cardiac involvement: case report and review of the literature. Am J Kidney Dis 2009;54:e9-15.

9. Nadrous HF, Yu AC, Specks U, et al. Pulmonary involvement in HenochSchönlein purpura. Mayo Clin Proc 2004; 79:1151-7.

10. Soloukides A, Moutzouris DA, Metaxatos G, et al. Pulmonary involvement in Henoch-Schonlein purpura. Emerg Med J 2006;23:886.

11. Toyooka T, Aizawa T, Suzuki N, et al. Increased plasma level of endothelin-1 and coronary spasm induction in patients with vasospastic angina pectoris. Circulation 1991;83:476-83.

12. Tsuda E, Yasuda T, Naito H. Vasospastic angina in Kawasaki disease. J Cardiol 2008;51:65-9
13. Buhaescu I, Williams A, Yood R. Rare manifestations of Churg-Strauss syndrome: coronary artery vasospasm, temporal artery vasculitis, and reversible monocular blindness-a case report. Clin Rheumatol 2009;28:231-3.

14. Tian Z, Zhang S, Wu Q. Recurrent variant angina pectoris due to Behçet's syndrome. Intern Med 2011;50:2587-9.

15. Shin JI, Lee JS. Severe coronary artery disease in Henoch-Schoenlein purpura: superimposition of antiphospholipid or polyangitis overlap syndrome? Int $\mathrm{J}$ Cardiol 2007;122:182-3.

16. Kardasz I, De Caterina R. Myocardial infarction with normal coronary arteries: a conundrum with multiple aetiologies and variable prognosis: an update. J Intern Med 2007;261:330-48.

17. Niaudet P, Habib R. Methylprednisolone pulse therapy in the treatment of severe forms of Schönlein-Henoch purpura nephritis. Pediatr Nephrol 1998;12:238-43.

18. Pozzi C, Bolasco PG, Fogazzi GB, et al. Corticosteroids in IgA nephropathy: a randomised controlled trial. Lancet 1999;353: 883-7. 